Delft University of Technology

\title{
Relocatable Energy Storage Systems for Congestion Management
}

Janssen, Suzanne ; Fu, Aihui; Cvetkovic, Milos; Palensky, Peter

DOI

10.1109/ISGT-Europe47291.2020.9248849

Publication date

2020

Document Version

Final published version

Published in

2020 IEEE PES Innovative Smart Grid Technologies Europe (ISGT-Europe)

\section{Citation (APA)}

Janssen, S., Fu, A., Cvetkovic, M., \& Palensky, P. (2020). Relocatable Energy Storage Systems for

Congestion Management. In 2020 IEEE PES Innovative Smart Grid Technologies Europe (ISGT-Europe): Proceedings (pp. 1166-1170). [9248849] IEEE . https://doi.org/10.1109/ISGT-Europe47291.2020.9248849

\section{Important note}

To cite this publication, please use the final published version (if applicable).

Please check the document version above.

\section{Copyright}

Other than for strictly personal use, it is not permitted to download, forward or distribute the text or part of it, without the consent of the author(s) and/or copyright holder(s), unless the work is under an open content license such as Creative Commons.

\section{Takedown policy}

Please contact us and provide details if you believe this document breaches copyrights.

We will remove access to the work immediately and investigate your claim. 
Green Open Access added to TU Delft Institutional Repository

'You share, we take care!' - Taverne project

https://www.openaccess.nl/en/you-share-we-take-care

Otherwise as indicated in the copyright section: the publisher is the copyright holder of this work and the author uses the Dutch legislation to make this work public. 


\title{
Relocatable Energy Storage Systems for Congestion Management
}

\author{
Suzanne Janssen, Aihui Fu, Miloš Cvetković, Peter Palensky \\ Intelligent Electrical Power Grids \\ Delft University of Technology \\ Delft, the Netherlands \\ suzanne.janssen.ee@gmail.com, a.fu@tudelft.nl, m.cvetkovic@tudelft.nl, p.palensky@tudelft.nl
}

\begin{abstract}
The rapid growth of rooftop solar, and on the rise technologies such as electric vehicles and heat pumps, is leading to congestion problems in low voltage distribution networks. If not dealt with, the congestion will prevent the installation of further units limiting the pace of the energy transition. Energy Storage Systems (ESS) are often seen as the technology with a high potential for congestion reduction. In this paper, we propose to use Relocatable Energy Storage System (RESS) fleet, to physically move the ESS throughout the distribution grid in an effort to alleviate congestion. The proposed method consists of two parts. First, a Mixed Integer Linear Program (MILP) is created to minimise the amount of RESS used and penalise displacement. Second, an algorithm with a Minimum Cost Maximum Matching objective is used to determine the dispatch of each individual RESS. The results on a CIGRE test system show the benefits of this method compared to stationary placed ESS in the grid.

Index Terms-Congestion Management, MILP, Energy Storage System
\end{abstract}

\section{INTRODUCTION}

In 2018 the Dutch government, businesses and other stakeholders started negotiating an agreement to combat climate change (Klimaatakkoord [1]). The outcome of this agreement is a target for the electricity production in the Netherlands, which establishes $70 \%$ of renewable generation by 2030 [2]. The ambition of this agreement puts challenges not only on energy production and consumption, but also on distribution and transmission of energy. A Dutch Distribution System Operator (DSO), Alliander, published an overview of physical grid locations where new initiatives, regarding production and consumption of electricity, could result in technical challenges [3], [4]. They state that increasing demand for energy intensive sectors causes capacity problems in the electricity grid. The method of congestion management based on market mechanisms will not suffice and companies will need to wait up to four years if they want to consume more electricity or deliver electricity to the grid.

The grid congestion can be resolved in different ways. For example, indirect control methods like day-ahead dynamic tariff and distribution capacity market [5] rely on market-based approaches to congestion alleviation. Direct control methods like reconfiguration and (re-)active power control give more certainty to the grid operator. Furthermore, Energy Storage Systems (ESSs) are often used as the adequate technology for providing ancillary service of congestion prevention. For example, battery energy systems are applied to reduce operational costs and grid tension coming from the charging stations of EVs [6]. Since the Electric Vehicles (EVs) are one of the main causes of congestion, considerable work has been put into orchestrating their behaviour to avoid congestion. In [7] and [8] EV fleet charging is optimised to regulate frequency and minimise the cost for charging the fleet. In [9], the EV charging is optimised to avoid congestion while satisfying driver's charging preferences. Indirect congestion control methods have been proposed with a similar intention, focusing on the price of electricity to spread out charging behaviour [10], [11].

The literature review shows that the distribution grid congestion elimination using ESS has so far been focused either on the stationary ESS in the grid or on the smart control of EV charging behaviour while respecting user preferences. In this paper, we explore the possibility of using a fleet of EVs (or more generally, a fleet of relocatable ESS) for the sole purpose of congestion management in the distribution grid. We call such fleet a Relocatable Energy Storage System (RESS) fleet. The added value, when compared to fixed ESS in the grid, is the opportunity of usage at different locations over time. The RESS have the possibility to move from one location to another following the congestion patterns. Compared to residential EVs, RESS can solely be used for congestion management, and as such, they could differ in size from EVs and they do not have to observe any unrelated preferences (such as the driving-habits of residential EV owners). In a rare study of relocatable ESS in the literature [12], a mobile battery energy storage is transported to different regions and kept stationary for longer periods of time (throughout entire seasons) in order to maintain reliability and power quality in the distribution system. In contrast to this reference, we look at relocation of ESS units on shorter time intervals (half-hourly) for the purpose of congestion management.

In this paper, a method is proposed to determine the optimal location for a RESS fleet over time for congestion prevention while minimising the number of RESS used. The DSO plays a crucial role, since it is responsible for electricity delivery and therefore congestion elimination. They are familiar with the topology of the grid and have insight in the production and demand in the grid, giving them the means to determine location where a RESS should be placed to prevent congestion. 
It is also in their benefit to minimise the number of RESS used as it minimises the cost of such service.

We further propose a method to optimally dispatch each vehicle in the RESS fleet to the determined location. The RESS fleet could be owned and operated by a DSO, but it could also be in the hands of a commercial organisation. Hence, the method for vehicle dispatch is intentionally created independent from the method for optimal location selection. The RESS vehicle dispatch method minimises the travel distance and the cost of travelling.

The paper is organised as follows. The methodology of determining for each time step where a RESS should be placed is explained in Section II. The method to determine which route an individual RESS should take is explained in section III. Section IV introduces the benchmark case of stationary ESS against which our method is compared. Section $\mathrm{V}$ introduces the test case and presents the simulation results. Section VI makes the conclusion.

\section{LOCATION OPTIMIZATION FOR RESS}

The first step in solving the congestion problem with RESS is to find optimal locations for the RESSs technology while minimising the amount of RESS needed. A Mixed Integer Linear Program (MILP) is used to determine these locations as described next.

\section{A. Objective function}

The objective of the optimization is to minimise the displacement over the entire considered time horizon and to make sure the required number of RESS is minimal. The displacement is minimized in order to avoid unnecessary relocation costs. This optimization problem is formulated using decision vector $x$ representing buses of the grid. The elements of this vector take value $x_{i}=1$ when a RESS is connected to bus $i$ and 0 otherwise. The first part of the objective function in (1) penalizes displacement. The second part penalizes the number of RESS. Choosing $\beta$ larger than $\alpha$ corresponds to the assumption that moving a vehicle is less expensive than engaging an extra vehicle.

$$
\min \alpha \sum_{t=1}^{T}|x(t)-x(t+1)|_{1}+\beta \sum_{t=1}^{T} \sum_{i=1}^{N} x_{i}(t)
$$

where:

$x(t)$ : binary vector of RESS locations at $t . x_{i}=1$ when a battery is connected to bus $i . x_{i}=0$ otherwise,

$\alpha, \beta$ : weighting constants (to make $x$ sparse, $\alpha$ should be smaller than $\beta$ ),

$N$ : the set of buses in the grid,

$T$ : time horizon,

$t \quad:$ time steps, $t=1,2,3 \ldots T$,

$i \quad$ : bus number, $i=1,2, \ldots, N$.

\section{B. Constraints}

The power balance, limitations of the maximum power flow on each line and limitations on the maximum generation output are included as the constraints. Equation (2) ensures that power supply equals power demand at all times. The line limits are modeled in Equation (3). This equation contains the matrix representation of DC power flow in which the line flows coming from RESS are included by superposition. Equation (4) makes sure that RESS operate within their available power constraints. Equations (5) relates the binary $x$-vector and the $\mathrm{P}$ vector of continuous RESS power injection values, i.e. $x_{i}=1$ when $P_{i} \neq 0$. This equation comes from using the Big $\mathrm{M}$ method.

$$
\begin{aligned}
& \text { s.t. } \quad \sum_{i=1}^{N} P_{i}(t)=-\sum_{i=1}^{N} P_{i, g r i d}(t) \quad \forall t
\end{aligned}
$$

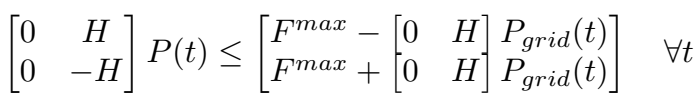

$$
\begin{aligned}
& P^{\min } \leq P(t) \leq P^{\max } \quad \forall t \\
& P(t) \leq M x(t) \quad \forall t \\
& -P(t) \leq M x(t) \quad \forall t
\end{aligned}
$$

$$
\begin{aligned}
& \text { where: } \quad \text { : vector of power injections of RESS at time } \\
& \text { t. } P_{i} \text { is the power injection at bus } i \text {, } \\
& P_{\text {grid }}(t) \quad \text { : vector of power injections other than from } \\
& \text { RESS (e.g. load) at time } t \text {, } \\
& F^{\max } \quad: \text { maximum flow at each line, } \\
& H \quad \text { : the distribution factor matrix, } \\
& P^{\min }, P^{\max }: \text { the minimum and maximum power limits } \\
& \text { of RESS, } \\
& M \quad \text { : big M, a very large number }
\end{aligned}
$$

Together, Equations (1) to (5) form the MILP for the optimization of location of RESS.

\section{Inclusion of power exchange with external parties}

In the optimisation (1) to (5), the RESS are optimized to avoid exchange of energy with the entities which do not belong to the RESS fleet. Equation (2) ensures this as the sum on the right-hand side of the equality sign is always zero. Hence, the net-power balance of all RESS injections is zero. In other words, RESS are only deployed to alleviate the congestion, while their total power balance is neutral. This assumption corresponds well with the regulatory requirements imposed on DSOs, according to which the DSOs are not allowed to supply or consume power. In this way, the power balance of other grid stakeholders is not altered.

In this subsection, we relax this assumption and allow netpower balance of RESS to be different than zero. In such case, we say that the RESS exchange power with external parties.

This relaxation is implemented in the model by allowing one element of power vector $P(t)$ to take values which are larger/smaller than the technical maximum/minimum for RESS injection. In particular, bus 0 is chosen. The new limits 
of vector $P$ are $P^{\max , 0}$ and $P^{\min , 0}$ and Equation (4) is replaced by Equation (6).

$$
P^{\min , 0} \leq P(t) \leq P^{\max , 0} \quad \forall t
$$

One implication of this relaxation is that the RESS fleet is now able to rely not only on its own units, but also on other players in the energy landscape. In other words, if the RESS fleet does not have sufficient capacity to relieve the congestion, it could team up with external parties, ensuring adequate outcome.

\section{DiSPATCH OF RESS FLEET}

In section II the optimal locations for RESS for each time step were determined for the entire time horizon $T$ by the grid operator. Whether a bus is occupied by a RESS is represented in vector $x(t)$.

In this section, we look at the dispatch problem of the fleet owner whose question is: what route should an individual RESS follow to minimise travel cost? To that end, the total number of RESS needed is calculated first. Then, the dispatch algorithm for each individual RESS is outlined.

In this paper, we assume that one time step is enough for a RESS to travel to its new location. For a specific real-world case, this assumption would have to be carefully evaluated and possibly adjusted since the duration of travel is correlated with the geo-spatial characteristics of the urban or rural environment, traffic patterns, weather conditions, etc.

The total number of required RESS can be expressed as the largest value over $t$ of the amount of nonzero elements in $x(t)+x(t+1)$. This is mathematically formulated as

$$
v=\max _{t} \operatorname{card}(x(t)+x(t+1))
$$

where the cardinality is defined as the amount of nonzero entries in the vector. Note that two successive time instances can contain the same amount of RESS, but at a different location. As a RESS cannot instantly appear at a new location, the number of required RESS units is calculated over two successive time instances.

Once the number of required RESS $(v)$ is known, planning of the dispatch of the RESS units can be made for each individual RESS. The planning algorithm consists of the following rules:

1) If $R E S S_{i}$ is connected to bus $n$ and at the next time step a RESS is needed at the same bus $n, R E S S_{i}$ will stay in its position. In time step $t+1$, bus $n$ is occupied.

2) If $R E S S_{i}$ is connected to bus $n$ and at the next time step no RESS is needed at bus $n, R E S S_{i}$ will go into a Free State, called state $F$.

3) If $R E S S_{i}$ is in state $F$, its previous location will be checked: $n$. If there exists a bus $n$ where a RESS needs to go to, $R E S S_{i}$ will remain at its position at bus $n$.

4) If in the next time step buses in $N_{t+1}^{*}$ are not occupied, an optimal decision will be made about which RESS in

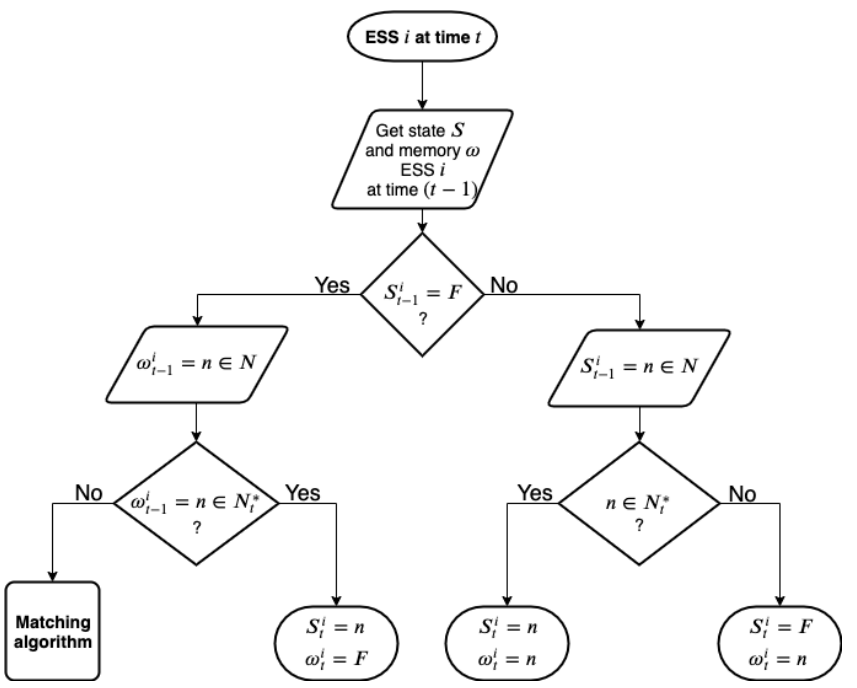

Fig. 1. Decision tree for one RESS. When all the RESS have been through the decision tree, the matching algorithm starts. $S_{t}^{i}$ is the state and $\omega_{t}^{i}$ a previous bus location.

state $F$ will go to which bus. This is achieved with the Matching Algorithm.

where:

$N \quad$ : set of buses with $n \in N$ as variable,

$N_{t}^{*} \quad:$ set of buses in the solution at t, $N_{t}^{*} \subseteq N$,

$F \quad$ : Free state, RESS is not connected to any bus,

$R E S S_{i}$ : Element of the set $\left[R E S S_{1}, \ldots, R E S S_{v}\right]$, the set of available RESS in the grid.

For a single RESS, the decision tree is shown in Figure 1.

The Matching Algorithm is used to decide the bus where the RESS in State $F$ should go. In the proposed Matching Algorithm, the total travelling costs of RESS is minimised. A full bipartite graph is constructed. The first set of nodes in the bipartition is the set of RESSs in the Free State, and the second set of nodes in the bipartition are the buses a RESS should travel to. The cost of an edge is similar to the travel distance between two nodes. Minimising the cost of the maximum matching means the total travel distance for the RESS is minimised. NetworkX [13] contains a package for manipulation of bipartite graphs. The minimum cost maximum matching algorithm from this package is used in this paper.

\section{STATIONARY ESS AS THE BENCHMARK CASE}

Stationary ESS case is used for comparison. In this case, the DSO places the stationary ESS at the most critical locations for congestion. We choose this case as the benchmark since this is one of the common approaches of the grid operators to deal with congestion.

In this case, the objective function changes as there is no displacement of the ESS. In the objective function, only the size of ESS is minimised accross all entries in $x$. As ESS are stationary, so the vector $x$ does not change over time, i.e. the vector $x$ is the same for all time steps. As a result, the MILP changes to: 


$$
\begin{aligned}
& \min \sum_{i=1}^{N} x_{i} \\
& \text { s.t. } \quad \sum_{i=1}^{N} P_{i}(t)=-\sum_{i=1}^{N} P_{i, g r i d}(t) \quad \forall t
\end{aligned}
$$

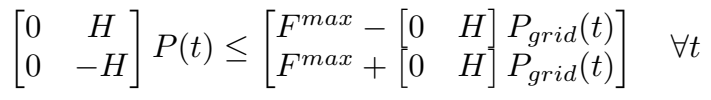

$$
\begin{aligned}
& P^{\min } \leq P(t) \leq P^{\max } \quad \forall t \\
& P(t) \leq M x \quad \forall t \\
& -P(t) \leq M x \quad \forall t
\end{aligned}
$$

As the ESS are statically placed, planning algorithm in section III is not used.

\section{Simulation Results}

\section{A. Test grid}

In this paper, the CIGRE low voltage distribution network (the Residential Branch) from [14] is used to test the proposed congestion management method. To that end, the thermal limits for maximum power flow on the power carriers are reduced to $400 \mathrm{~kW}$. The time step of the algorithm is chosen to be 30 minutes, as this was found to be an appropriate time for the grid of this size: the estimated, maximum time for a vehicle to disconnect, move a maximum distance within the grid and reconnect. Each bus contains from 5 up to 200 households. The power consumption profile is obtained from [15]. The power production profile for solar energy is obtained from [16]. These injections are considered deterministic by the proposed method. Hence, the power consumption of residents and the power production of solar panels are perfectly known.

The characteristics of the RESSs are assumed as follows:

1) Power consumption while relocating: $0.1 \mathrm{kWh} / 5 \mathrm{~km}$.

2) Maximum power output: $120 \mathrm{~kW}$ (fast charging).

3) Minimum power output: $-120 \mathrm{~kW}$.

4) Energy capacity: $85 \mathrm{kWh}$, though the SOC is not optimised for.

\section{B. Results}

First, power flow is simulated in the test network, and Figure 2 illustrates the total amount of congested lines per time step. The congestion peak occurs during the day between 11am and $5 \mathrm{pm}$ when the generation of solar power is high.

In order to solve the congestion problem, we perform the following simulations:

1) Case I: Optimization of RESS fleet location and dispatch

2) Case II: Optimization of RESS fleet location and dispatch including power exchange with external parties

3) Case III: Benchmark case of stationary ESS

The results of Case I are shown in Figure 3. The results show that 5 RESS are required in total. To improve visibility, the starting positions are not represented and only the last twelve hours of the day are shown. The results present the

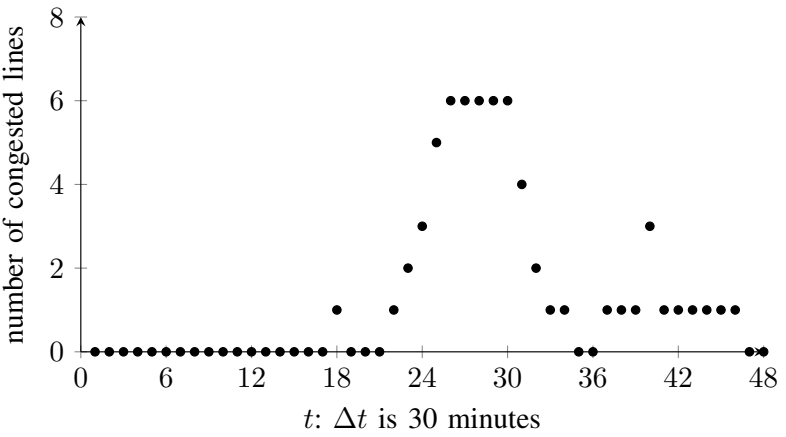

Fig. 2. Amount of congested lines per time step (each step is $30 \mathrm{~min}$ long and the $\mathrm{x}$-axis shows one day).

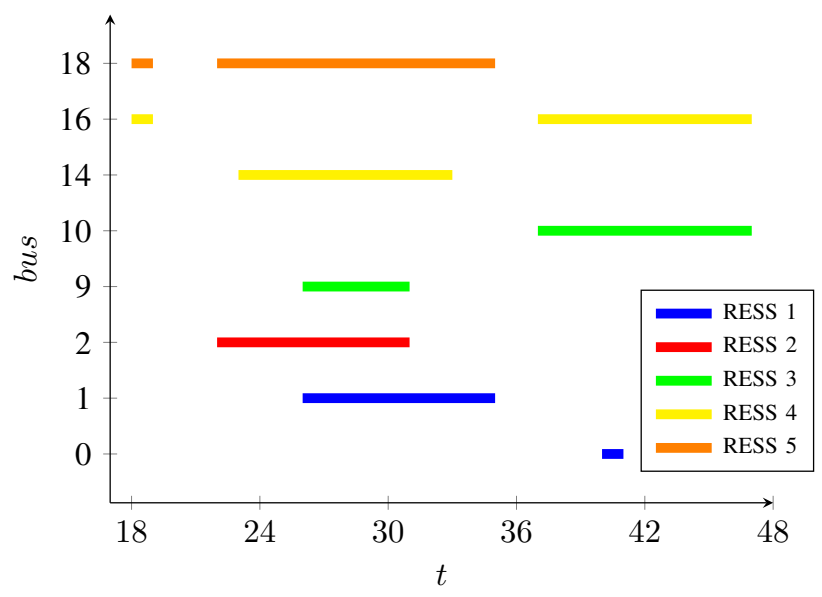

Fig. 3. Case I: Optimization of RESS fleet location and dispatch.

location of RESS at each time, and the different colours denote individual RESSs in order to give insight into their travel route.

If RESS are allowed to exchange power with external parties, such is the Case II, the simulation results change considerably (see Figure 4). In this case, 3 RESS are required in total, which is quite lower compared to Case I. This is an expected result since RESS are not constrained to achieve net-zero sum of their power injections.

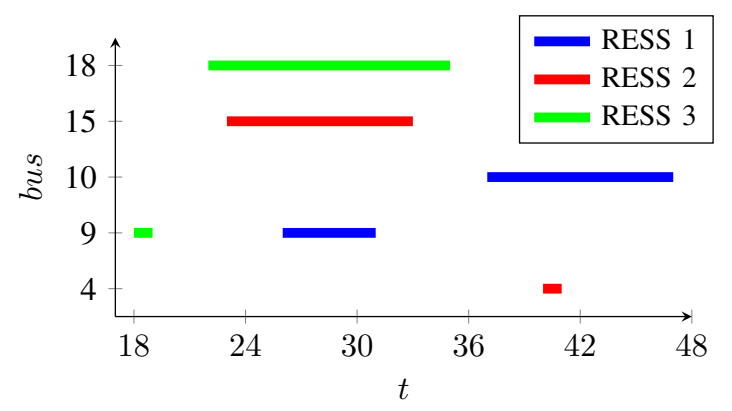

Fig. 4. Case II: Optimization of RESS fleet location and dispatch including power exchange with external parties. 


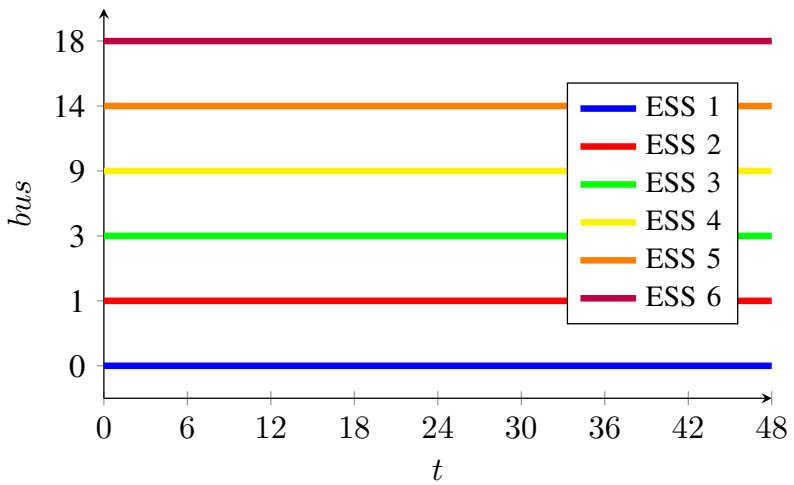

Fig. 5. Case III: Benchmark case of stationary ESS.

Case III is shown for comparison. In this case, stationary ESS is used. In total, 6 ESS are required, and they stay at their position for the entire day. Figure 5 shows the position of each ESS. Compared with Case I, one more ESS is needed, and compared with Case II, three more ESS are needed. This result shows that the RESS fleet has a higher efficiency and utilization than statically placed ESS for solving the congestion problem.

\section{Discussion}

The methodology for congestion prevention with RESS was achieved by splitting up the problem in two parts. The motivation for this partitioning is found in the regulations imposed on DSOs in deregulated electricity sectors. This partition is not necessary in the case of the vertically integrated utilities. In such environment, the DSO is allowed to assume full control over the RESS fleet and the methodology suggested in this paper could be integrated and further improved.

Our model simplifies the connection requirements for RESS. It is assumed that each node could host a RESS and that no more than one RESS is connected to any one node at a time. The first part of this assumption enlarges the solution space while the second one shrinks it. Exploring the implications of the assumption could lead to further discoveries.

The correlation of the results with the size of the grid, traffic, weather conditions, geo-spatial characteristics of the area, etc. is one of the aspects which will be explored in future work.

\section{CONCLUSION}

This paper proposed a method to optimally distribute RESS in order to prevent congestion in the grid. The method is split in two parts. The first part determines the optimal locations of RESS fleet in order to reduce the congestion level and is solved by the grid operator. The second part is a planning algorithm to determine the route individual RESS, operated by the fleet operator, should travel to reach the given locations. Bipartite matching is used to develop the optimal route. The results show that the use of relocatable ESS is preferable over stationary ESS for the chosen case scenario. If the RESS are allowed to exchange the energy with the entities outside of their fleet, their benefits further increase.

\section{REFERENCES}

[1] Dutch Government, Klimaatakkoord.nl, 2019.

[2] —, Klimaatakkoord elektriciteit, 2019.

[3] Liander, Congestiemanagement: onderzoek en resultaat, 2019.

[4] _ Liander actualiseert knelpunten in het elektriciteitsnet, 2019.

[5] S. Huang, Q. Wu, Z. Liu, and A. H. Nielsen, "Review of congestion management methods for distribution networks with high penetration of distributed energy resources," in IEEE PES Innovative Smart Grid Technologies, Europe, IEEE, 2014, pp. 1-6.

[6] M. Gjelaj, C. Traeholt, S. Hashemi, and P. B. Andersen, "DC Fast-charging stations for EVs controlled by a local battery storage in low voltage grids," in 2017 IEEE Manchester PowerTech, 2017, pp. 1-6.

[7] N. DeForest, J. S. MacDonald, and D. R. Black, "Day ahead optimization of an electric vehicle fleet providing ancillary services in the Los Angeles Air Force Base vehicle-to-grid demonstration," Applied Energy, vol. 210, pp. 987-1001, 2018, ISSN: 03062619.

[8] O. Sundström and C. Binding, "Optimization Methods to Plan the Charging of Electric Vehicle Fleets," in Proc. of the international conference on control, communication and power engineering, 2010, pp. 28-29.

[9] _ - "Flexible charging optimization for electric vehicles considering distribution grid constraints," IEEE Tran on Smart Grid, vol. 3, no. 1, pp. 26-37, 2012.

[10] N. O'Connell, Q. Wu, J. Østergaard, A. H. Nielsen, S. T. Cha, and Y. Ding, "Day-ahead tariffs for the alleviation of distribution grid congestion from electric vehicles," Electric Power Systems Research, vol. 92, pp. 106-114, 2012.

[11] J. Hu, S. You, M. Lind, and J. Østergaard, "Coordinated charging of electric vehicles for congestion prevention in the distribution grid," IEEE Transactions on Smart Grid, vol. 5, no. 2, pp. 703-711, 2014, ISSN: 19493053.

[12] Y. Zheng, Z. Dong, S. Huang, K. Meng, F. Luo, J. Huang, and D. Hill, "Optimal integration of mobile battery energy storage in distribution system with renewables," Journal of Modern Power Systems and Clean Energy, vol. 3, no. 4, pp. 589-596, 2015.

[13] A. A. Hagberg, D. A. Schult, and P. J. Swart, "Exploring Network Structure, Dynamics, and Function using NetworkX," in Proceedings of the 7th Python in Science Conference, G. Varoquaux, T. Vaught, and J. Millman, Eds., Pasadena, CA USA, 2008, pp. 11-15.

[14] K. Strunz, C. Abbey, C. Andrieu, R. C. Campbell, and R. Fletcher, Benchmark Systems for Network Integration of Renewable and Distributed Energy Resources. 2009.

[15] U. P. Networks, SmartMeter Energy Consumption Data in London Households, 2013.

[16] Elia Group, Generation. 